\title{
Formation and Growth of Silver Nanocubes upon Nanosecond Pulsed Laser Irradiation: Effects of Laser Intensity and Irradiation Time
}

\author{
Umair Yaqub Qazi' ${ }^{1}$ Zameer Shervani ${ }^{2 *}$, Rahat Javaid ${ }^{3}$, Shinji Kajimoto4, Hiroshi Fukumura4 \\ ${ }^{1}$ Division of Nanomaterials and Chemistry, Hefei National Laboratory for Physical Sciences at Microscale, \\ University of Science and Technology of China, Hefei, China \\ ${ }^{2}$ Nanomaterials Production Division, Food and Energy Security Research and Product Center, Sendai, Japan \\ ${ }^{3}$ Fukushima Renewable Energy Institute, National Institute of Advanced Industrial Science and Technology, \\ AIST, Fukushima, Japan \\ ${ }^{4}$ Department of Chemistry, Graduate School of Science, Tohoku University, Sendai, Japan \\ Email: *shervani.nanotek@gmail.com
}

How to cite this paper: Qazi, U.Y., Shervani, Z., Javaid, R., Kajimoto, S. and Fukumura, H. (2017) Formation and Growth of Silver Nanocubes upon Nanosecond Pulsed Laser Irradiation: Effects of Laser Intensity and Irradiation Time. Advances in Nanoparticles, 6, 148-157.

https://doi.org/10.4236/anp.2017.64013

Received: September 22, 2017

Accepted: November 3, 2017

Published: November 6, 2017

Copyright $\odot 2017$ by authors and Scientific Research Publishing Inc. This work is licensed under the Creative Commons Attribution International License (CC BY 4.0).

http://creativecommons.org/licenses/by/4.0/

\begin{abstract}
Silver nanoparticles (AgNPs) were fabricated by repetitive irradiation of near ultraviolet (UV) nanosecond laser pulses (355 nm, $5 \mathrm{~ns}$ ) in an aqueous solution of silver nitrate in the absence of stabilizing agents. A broad absorption peak was observed in the visible region showing the formation of a variety of AgNPs in the solution. Among the variety of products, it was found that silver nanocubes (AgNCs) grew in size with longer laser irradiation time. The size of AgNCs also increased with higher laser intensity. The average size of AgNCs, investigated by a scanning electron microscope (SEM) was in the range of 75 $200 \mathrm{~nm}$. The number of reduced atoms in AgNCs as a function of laser intensity showed that the AgNCs are apparently produced by a four photon process, implying that the formation of dimer silver atoms is essential for the formation.
\end{abstract}

\section{Keywords}

Nanosecond Laser, Silver Nanocubes, Nanoparticle Synthesis, Laser Intensity, Irradiation Time

\section{Introduction}

In last few decades, fabrication of nanomaterials (NMs) has been of great interest because of their unique physicochemical properties which differentiate them from those of the corresponding "bulk" materials [1] [2]. The optical and electronic 
properties of these NMs energetically change their attitude in assistance towards potential applications [3] [4] such as photovoltaic devices, catalysts, semiconductors, removal of toxic materials, medical diagnosis and pharmaceutical products [5] [6] [7]. Since physicochemical properties of NPs highly depend on their shape and size [8], thus the control of their shape and size is an important parameter. Noble metal NPs gained much attention due to their shape and size dependent properties such as optical, electrochemical, magnetic and catalytic properties [9] [10]. Among all other noble metals, AgNPs are suitable for various applications in different fields [11] [12]. As AgNPs have already proved to be a good candidate against bacteria, viruses and other microorganisms [13], they have much potential in the field of food preservation, public hygiene and medical care.

It has been challenging to adopt a suitable method for synthesizing shape and size controlled metal NPs [14] [15] [16]. Synthesis of NPs in a liquid medium can be done in different ways such as chemical, electrochemical and laser-based synthesis methods. From an environmental point of view, it is clear that laser based methods such as laser ablation and laser irradiation have advantages over others. This is because without toxic chemicals, it provides us a unique opportunity to obtain additive-free pure NPs suspension. Furthermore, different morphological changes can also be controlled by laser intensity, spot size, pulse width, wavelength and irradiation time. A limited study, however, has been reported on the synthesis of AgNPs in which photochemical reduction by pulsed laser irradiation is employed. A method using pulsed laser irradiation might be similar to radiolysis as photon works as a reducing agent, which provides electrons, and stable NPs are synthesized in an aqueous solution of metal salts containing suitable dispersing agents [17] [18] [19]. This method also helps us to generate metal NPs with narrow size distribution and completely free from extra reducing chemicals.

In a previous report [17], we showed that irradiation of a non-focused nanosecond near-UV laser pulse into an aqueous solution of silver salt induces biphotonic reduction of silver ions, leading to the formation of AgNPs. In that study, we also showed that Ag NPs, including NCs, formed with the laser-irradiation into silver nitrate solution without any additives, whereas nanospheres (NSs) having an average diameter of $14 \mathrm{~nm}$ were obtained when sodium dodecyl sulfate (SDS) was added into the solution before laser irradiation. In this study, we focus on the formation and growth of AgNCs upon nanosecond laser irradiation. We found that larger AgNCs were obtained with longer irradiation time. This result indicates that AgNCs grew with repetitive irradiation of UV pulses. The size of NCs also increased with higher laser intensity. The total volume of AgNCs was proportional to the 4th power of laser intensity. Thus we consider that the AgNC is apparently produced by a four photon process and the formation of dimer silver atoms is essential for the formation and growth of AgNCs. 


\section{Experimental}

Silver nitrate $\left(\mathrm{AgNO}_{3}\right.$, purity: 99.9\%) was purchased from Wako pure chemical industries, Ltd., and used without further treatment. A $10^{-3} \mathrm{M}$ aqueous solution of silver ions was prepared by dissolving $\mathrm{AgNO}_{3}$ in ultrapure water (Millipore, Simplicity UV) without adding any additives. A quartz cell $\left(10 \times 10 \times 45 \mathrm{~mm}^{3}\right)$ was used for laser-irradiation experiments and spectrum measurements. The volume of $\mathrm{AgNO}_{3}$ solution ( $3.5 \mathrm{ml}$ ) in the quartz cell was kept the same for all experiments. Figure 1 shows a schematic diagram of the experimental setup for laser irradiation. The third harmonic of Q-switched Nd: YAG laser (Quantel, Brilliant Nd: YAG, $355 \mathrm{~nm}, 6 \mathrm{~ns}, 10 \mathrm{~Hz}$ ) was introduced into the sample solution in the cell without using any focus lens. During laser irradiation, the sample solution was stirred continuously using a magnetic stirrer. All experiments were performed at room temperature $(295 \mathrm{~K})$ and 1 atmosphere pressure. The optical characteristics of the sample solutions before and after irradiation were analyzed using UV-visible spectrophotometer (Shimadzu UV-1600 PC). A small portion of the solution after laser irradiation was dropcast on indium titanium oxide (ITO) substrates and dried in a desiccator. Then, these substrates were observed using a scanning electron microscope (SEM, Hitachi, FE-SEM-S4300) for structural characterization of AgNPs formed in the solution.

\section{Results and Discussion}

Figure 2 shows UV-visible absorption spectra of aqueous solutions of $\mathrm{AgNO}_{3}$ before and after pulsed laser irradiation. The absorption peak at $302 \mathrm{~nm}$ is due to the presence of nitrate ions in the solution. Freshly prepared $\mathrm{AgNO}_{3}$ solution did not show absorption at 355 nor $405 \mathrm{~nm}$; however, when the sample solution was irradiated with ns laser pulses, a new broad absorption peak was observed at 405 $\mathrm{nm}$, indicating the formation of AgNPs which has the surface plasmon resonance (SPR) band around $400 \mathrm{~nm}$. The position and shape of the SPR band of
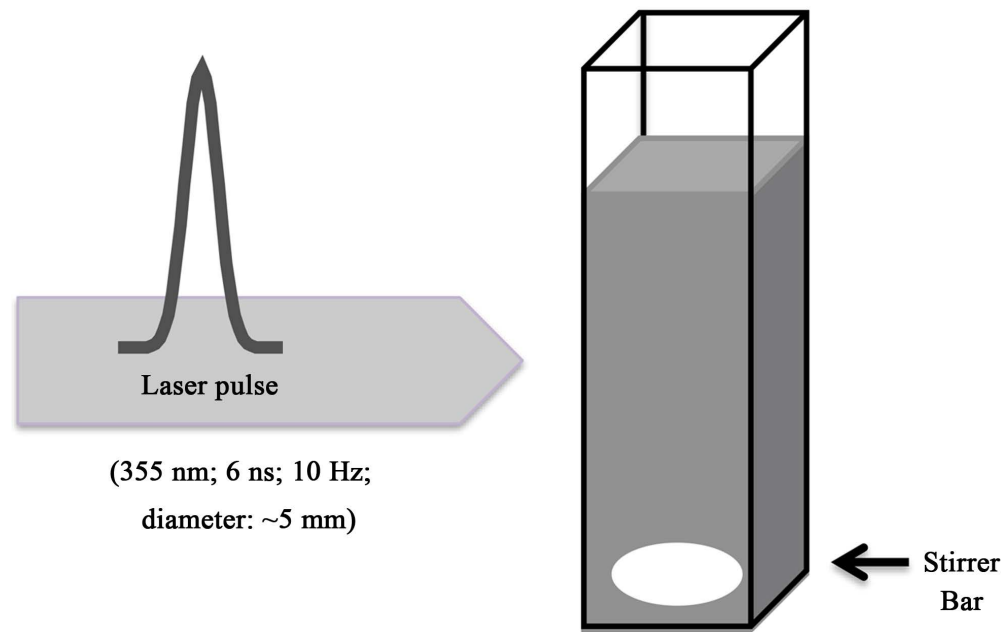

Figure 1. Experimental arrangement for fabrication of AgNPs in an aqueous solution of $\mathrm{AgNO}_{3}$ using nanosecond pulsed laser irradiation. 


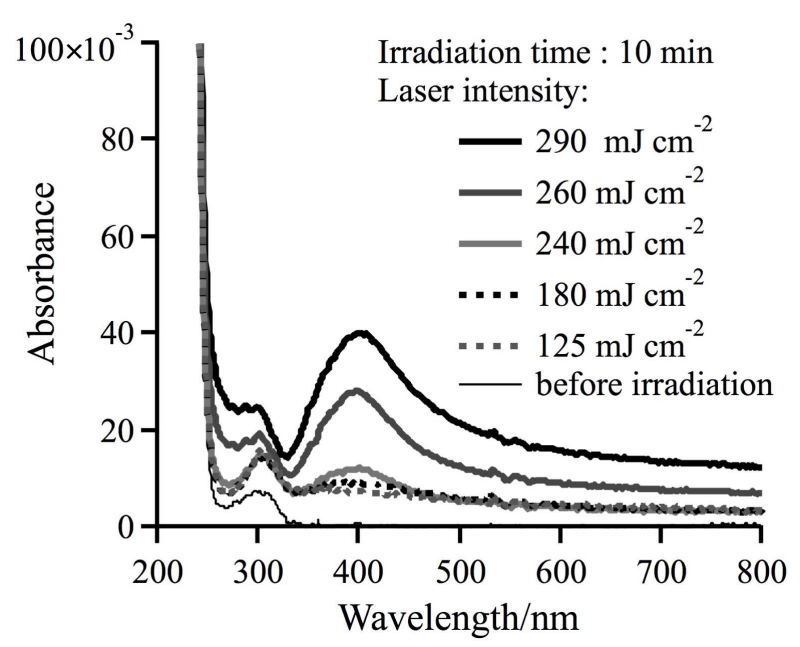

(a)

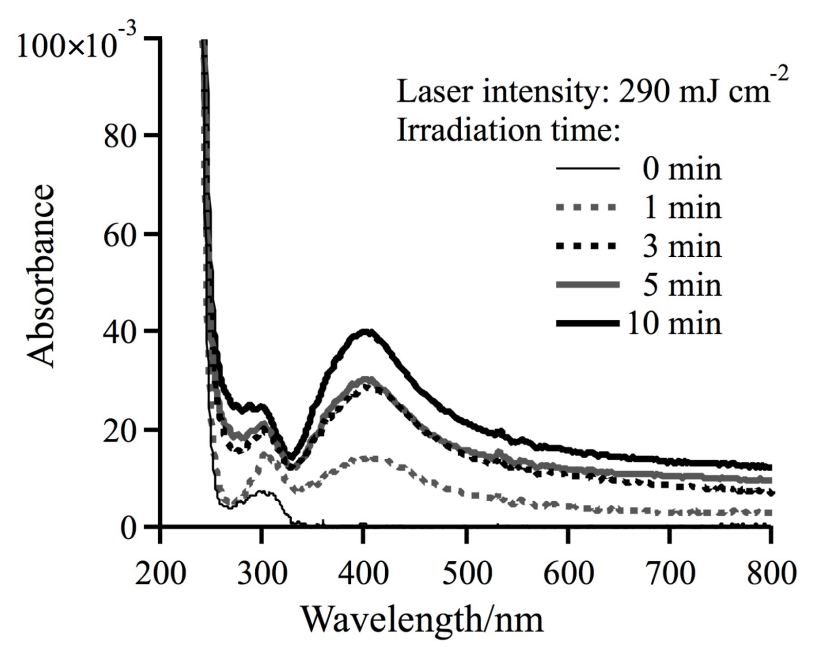

(b)

Figure 2. Absorption spectra of $\mathrm{AgNO}_{3}$ solution before and after ns pulsed laser irradiation: (a) effect of laser intensity at a fixed irradiation time of $10 \mathrm{~min}$; (b) effect of laser irradiation time at a fixed laser intensity of $290 \mathrm{~mJ} / \mathrm{cm}^{2}$.

noble metal NPs are strongly dependent on the particle size, dielectric medium and surface absorbed species [20] [21]. As shown in Figure 2, the peak was broad and has a tail toward longer wavelength, which indicates the formation of AgNPs having a wide range of shape and size distribution and the aggregation of products in the solution [19]. As Figure 2(a) shows, the higher the laser intensity was, the higher the absorbance of the solution became. This result agrees with our previous report [17]. Since the absorption of silver nitrate solution at $355 \mathrm{~nm}$ is little, the initial process can be two photon ionization of water leading to the ejection of electrons. With higher laser intensity, the yield of electrons will became higher and the number of reduced Ag atoms increased. The absorbance of products obtained after $10 \mathrm{~min}$ irradiation, however, was not proportional to the square of laser intensity. This can be mainly due to two reasons. The first reason arises from the heterogeneity of products. The size and structure distri- 
bution of products causes spectral inhomogeneity and makes a quantitative analysis of absorption spectra difficult. Another reason is due to the absorption of $355 \mathrm{~nm}$ laser pulses by products preformed with former pulses. As shown in Figure 2, products have absorbance at $355 \mathrm{~nm}$. The repetitive irradiation of UV pulses causes the excitation of silver nanoparticles, which can lead to electron ejection from nanoparticles and further formation and aggregation/growth of nanoparticles as well as the laser ablation of nanoparticles. These variety of processes would be no longer biphotonic process. The size and structure change by repetitive irradiation also causes spectral changes of products such that the peak absorbance is not proportional to photo-reduced Ag atoms nor to the square of laser intensity.

The formation and growth of AgNPs as a result of photo-reduction of $\mathrm{Ag}^{+}$ ions was also confirmed with SEM images. Figure 3 shows SEM images of products obtained after $10 \mathrm{~min}$ UV laser irradiation with different intensities. The synthesized photo-products had a broad shape and size distribution along with the formation of cubic, irregular and spherical AgNPs. Among these AgNPs, we studied cubic shaped NPs in detail in this study. A series of experiments was performed with varying the laser intensity and the irradiation time, and the size distribution of synthesised AgNCs was obtained for each experimental condition.

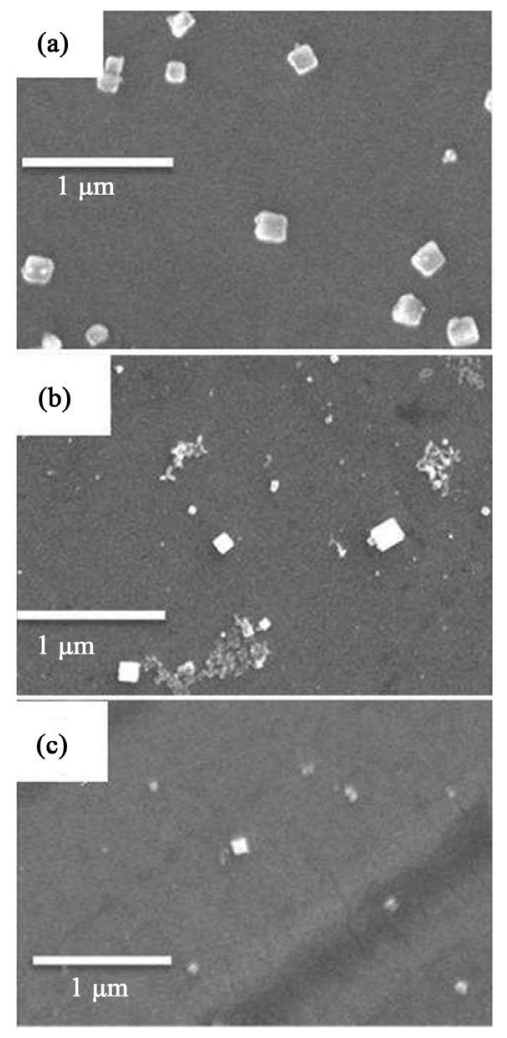

Figure 3. SEM images of Ag NCs, synthesized at various laser intensities at a fixed irradiation time of $10 \mathrm{~min}$ : (a) higher intensity $\left(290 \mathrm{~mJ} / \mathrm{cm}^{2}\right)$; (b) middle intensity $(240$ $\left.\mathrm{mJ} / \mathrm{cm}^{2}\right)$; and (c) lower intensity $\left(140 \mathrm{~mJ} / \mathrm{cm}^{2}\right)$. 
Figure 4 shows the size distribution of AgNCs obtained after 10 min UV laser irradiation with different intensities. The results can be categorized into three parts on the bases of the laser intensity i.e., (a) higher $\left[260-290 \mathrm{~mJ} \cdot \mathrm{cm}^{-2}\right]$, (b) middle $\left[180-240 \mathrm{~mJ} \cdot \mathrm{cm}^{-2}\right]$ and (c) lower intensity $\left[125-140 \mathrm{~mJ} \cdot \mathrm{cm}^{-2}\right]$. With lower laser intensity, few numbers of small Ag NCs were obtained. With middle laser intensity, the number of AgNC increased, whereas the average size stayed small; i.e. $90 \mathrm{~nm}$ for $180 \mathrm{~mJ} \cdot \mathrm{cm}^{-2}$ and $120 \mathrm{~nm}$ for $240 \mathrm{~mJ} \cdot \mathrm{cm}^{-2}$. With higher laser intensity, the number of AgNC increased and the averaged size became larger and larger; i.e. $180 \mathrm{~nm}$ for $260 \mathrm{~mJ} \cdot \mathrm{cm}^{-2}$ and $220 \mathrm{~nm}$ for $290 \mathrm{~mJ} \cdot \mathrm{cm}^{-2}$. These results clearly show that higher laser intensity gives the larger AgNCs and the number of products also increases with laser intensity. These results are also consistent with absorption spectra of products shown in Figure 2.

To investigate the growth process of AgNCs, we analysed the size distribution of AgNCs at different irradiation times. Figure 5 shows the time evolution of size distribution of Ag NC with the middle $\left(180 \mathrm{~mJ} \cdot \mathrm{cm}^{-2}\right)$ and higher laser intensity $\left(290 \mathrm{~mJ} \cdot \mathrm{cm}^{-2}\right)$. With middle laser intensity, the number of AgNCs obtained increased with longer irradiation time, whereas the averaged size stayed almost constant at $80-90 \mathrm{~nm}$. This means AgNCs formed but only slightly grew in size by further irradiation of UV laser pulses with middle intensity. On the other hand, the results with higher laser intensity were very different. With 290 $\mathrm{mJ} \cdot \mathrm{cm}^{-2}$ laser irradiation, AgNCs having the size less than $100 \mathrm{~nm}$ were obtained mainly with short irradiation time, similar to the result after long time irradiation with middle laser intensity. As the irradiation time increased, the number of

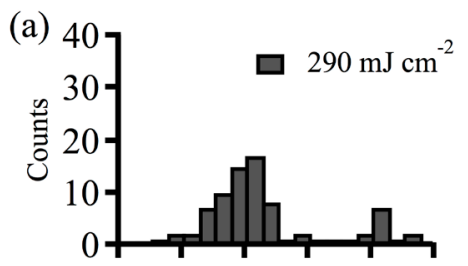

(c)
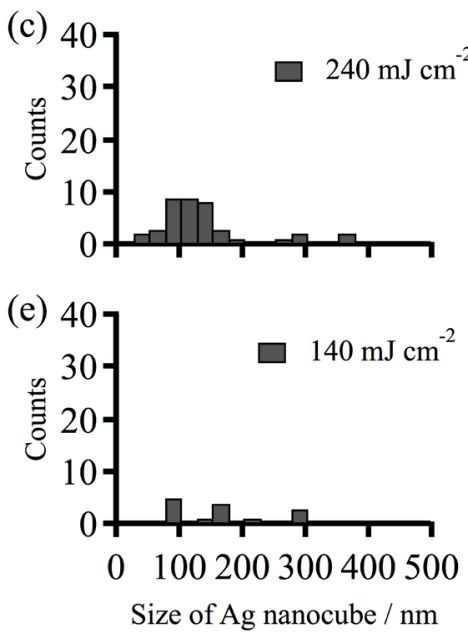

(b)

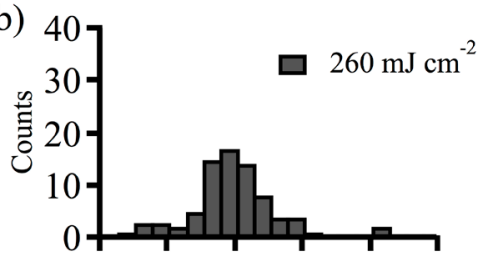

(d)

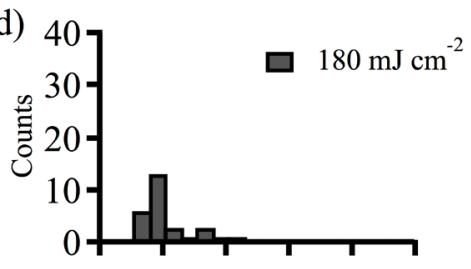

(f)

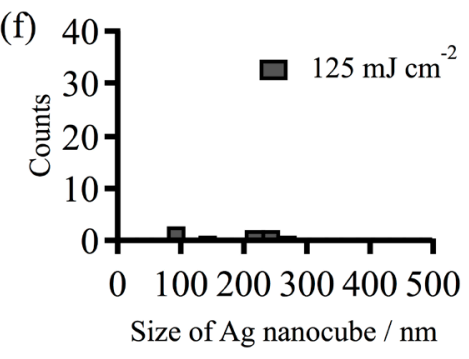

Figure 4. Effect of laser intensities on size distribution of AgNCs at a fixed irradiation time of 10 min: (a) 290; (b) 260; (c) 240 and (d) 180; (e) 140; (f) $125 \mathrm{~mJ} \cdot \mathrm{cm}^{-2}$. 


$$
\text { (a) }
$$

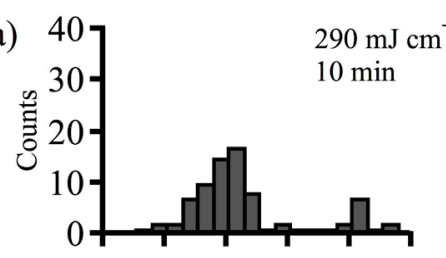

(b)

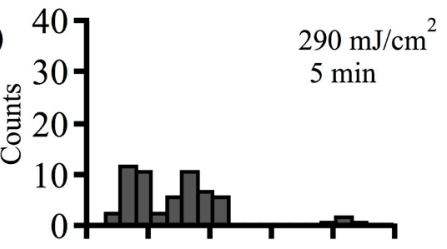

(c)

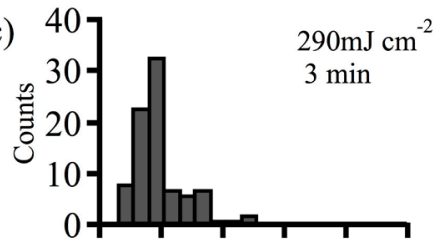

(d)

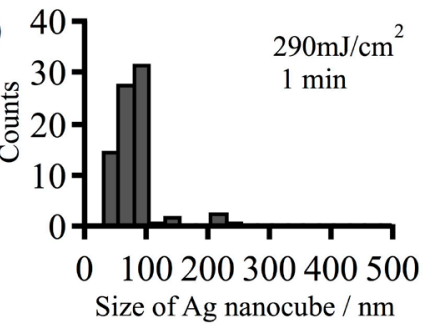

(e) $207 \quad 180 \mathrm{~mJ} \mathrm{~cm} \mathrm{~cm}^{-2}$

$10 \min$

(f)

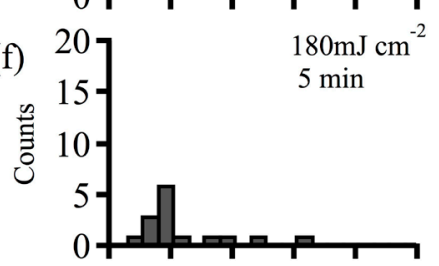

(g) $207 \quad 180 \mathrm{~mJ} \mathrm{~cm}^{-2}$

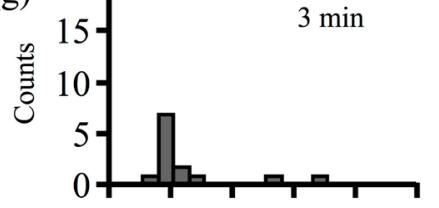

(h)

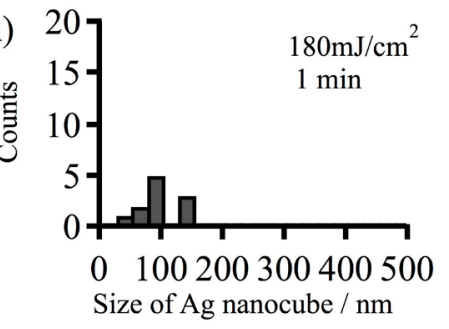

Figure 5. Effect of laser irradiation time on size distribution of AgNCs at $290 \mathrm{~mJ} \cdot \mathrm{cm}^{-2}$ (a)-(d) and $180 \mathrm{~mJ} \cdot \mathrm{cm}^{-2}$.

AgNCs less than $100 \mathrm{~nm}$ decreased and the number of larger AgNCs increased, resulting in the peak shift in the size distribution diagram shown in Figure 5(a) to Figure 5(d). These results clearly indicate that AgNCs grow with further repetitive irradiation of UV laser pulses and the size of AgNCs can be controlled by changing the laser intensity and irradiation time. Note that, we used a conventional quartz cell and a stirrer bar for the laser irradiation experiments, such that photoproducts formed with a former laser pulse can be irradiated with UV pulses repeatedly. This further pulse irradiation can cause the growth of AgNCs.

For the growth mechanism, there are two possible processes. One is via the direct excitation of previously formed AgNCs, which can induce electron ejection from AgNCs and/or reduction of Ag ions on the surface of AgNCs. The other is via bi-photonic process of water, which induces electron ejection. Ejected electrons would be easily captured by silver ions in solution and reduced silver atoms may be adsorbed on the AgNCs. In this case, during the formation process and also growth process, the source of electron is always water molecules excited by two photon process [17]. To understand the mechanism further, we plotted the total volume of AgNCs as a function of laser power as shown in Figure 6. The total volume was calculated from the product of the average size and the number of AgNCs counted. According to Figure 6, the total volume of products observed in a certain area is proportional to the 4 th power of the intensity 


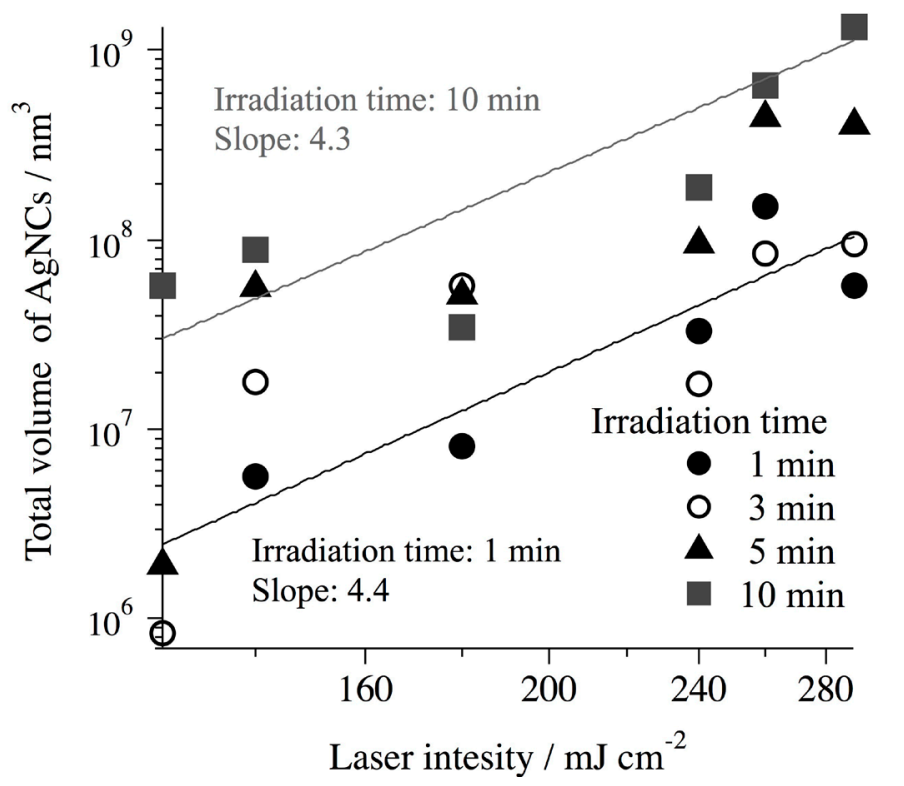

Figure 6. Effect of laser intensity on the total volume of AgNCs at different irradiation time.

of laser pulses. Here short irradiation (for example $1 \mathrm{~min}$ ) would have less effect of previously produced Ag NCs, while long-time irradiation (for example 10 $\min$ ) can be influenced by previously formed products. If long lifetime photoproducts including previously produced AgNCs reabsorbs laser light and reduce silver ions, this would be mostly one or two photon process and the intensity dependency slope should be less than four, which is inconsistent with the experimental result.

The apparent four-photon process observed here can be explained with the encounter of two silver atoms forming silver dimers as essential parts of the formation of NCs. Such kinetics clearly requires the dependency of products proportional to the square of the concentration of intermediates as shown in delayed florescence intensity induced by collision of two lowest-excited triplet states [22]. Because silver atom itself is yielded from solvated electrons generated by two photon process [17], overall intensity dependence is considered to be four-photon process as shown in the following scheme.

$$
\begin{gathered}
\mathrm{H}_{2} \mathrm{O}+2 h v \rightarrow \mathrm{H}_{2} \mathrm{O}^{+}+\mathrm{e}_{e q}^{-} \\
\mathrm{Ag}^{+}+\mathrm{e}_{a q}^{-} \rightarrow \mathrm{Ag}^{0} \\
\mathrm{Ag}^{0}+\mathrm{SDS} \rightarrow \text { Sphere (two photon formation) } \\
\mathrm{Ag}^{0}+\mathrm{Ag}^{0} \rightarrow \mathrm{Ag}^{0}{ }_{2} \text { (dimer) } \rightarrow \text { Cube (four photon formation) }
\end{gathered}
$$

Assuming that solvated silver atoms and dimers have certain lifetimes and limited diffusion constants, we can presume that these atoms or dimers can't form bigger AgNCs at a low concentration but may grow larger at a high concentration. This kind of kinetics may explain the Ag NCs' production process depending on the laser intensity. 


\section{Conclusion}

In this study, we demonstrated that AgNP production from pure $\mathrm{AgNO}_{3}$ aqueous solution, using direct laser irradiation, which differs from other laser processes such as the laser ablation of a solid silver target immersed in a liquid. Photo-reduction of $\mathrm{Ag}^{+}$ions was initiated by UV laser pulse $(355 \mathrm{~nm})$ and $\mathrm{Ag}$ NPs were obtained without using any additives. The size of Ag NCs varied with laser intensity and irradiation time. The size distribution obtained with different laser intensity and irradiation time revealed that the growth process of AgNCs was highly depended on these two parameters. The laser intensity dependence of the growth process suggests that the AgNC is apparently produced by four photon process. By controlling these parameters, we can synthesize additive-free metal nanoparticles with controllable size and shape.

\section{Acknowledgements}

UYQ is grateful to Watanuki International Scholarships Foundation for granting scholarship. This work was partly supported by JSPS KAKENHI Grant Numbers JP26286004 and JP15H03518.

\section{References}

[1] Pileni, M.P. (1997) Nanosized Particles Made in Colloidal Assemblies. Langmuir, 13, 3266-3276. https://doi.org/10.1021/la960319q

[2] Henglein, A. (1989) Small-Particle Research: Physicochemical Properties of Extremely Small Colloidal Metal and Semiconductor Particles. Chemical Reviews, 89, 1861-1876. https://doi.org/10.1021/cr00098a010

[3] Linsebigler, A.L., Lu, G. and Yates, J.T. (1995) Photocatalysis on $\mathrm{TiO}_{2}$ Surfaces: Principles, Mechanisms, and Selected Results. Chemical Reviews, 95, 735-758. https://doi.org/10.1021/cr00035a013

[4] Fox, M.A. and Dulay, M.T. (1993) Heterogeneous Photocatalysis. Chemical Reviews, 93, 341-357. https://doi.org/10.1021/cr00017a016

[5] Sharma, S., Khawaja, M., Ram, M.K., Goswami, D.Y. and Stefanakos, E. (2014) Characterization of 10, 12-Pentacosadiynoic Acid Langmuir-Mlodgett Monolayers and Their Use in Metal Insulator-Metal Tunnel Devices. Beilstein Journal of Nanotechnology, 5, 2240-2247. https://doi.org/10.3762/bjnano.5.233

[6] Arooj, S., Nazir, S., Nadhman, A., Ahmad, N., Muhammad, B., Ahmad, I., Mazhar, K. and Abbasi, R. (2015) Novel ZnO:Ag Nanocomposites Induce Significant Oxidative Stress in Human Fibroblast Malignant Melanoma (Ht144) Cells. Beilstein Journal of Nanotechnology, 6, 570-582. https://doi.org/10.3762/bjnano.6.59

[7] Guo, L., Jing, D., Liu, M., Chen, Y., Shen, S., Shi, J. and Zhang, K. (2014) Functionalized Nanostructures for Enhanced Photocatalytic Performance under Solar Light. Beilstein Journal of Nanotechnology, 5, 994-1004.

https://doi.org/10.3762/bjnano.5.113

[8] Rycenga, M., Cobley, C.M., Zeng, J., Li, W., Moran, C.H., Zhang, Q., Qin, D. and Xia, Y. (2011) Controlling the Synthesis and Assembly of Silver Nanostructures for Plasmonic Applications. Chemical Reviews, 111, 3669-3712. https://doi.org/10.1021/cr100275d 
[9] Mulvaney, P., Linnert, T. and Henglein, A. (1991) Surface Chemistry of Colloidal Silver in Aqueous Solution: Observations on Chemisorption and Reactivity. The Journal of Physical Chemistry, 95, 7843-7846. https://doi.org/10.1021/j100173a053

[10] Feng, X., Cheng, Y., Ye, C., Ye, J., Peng, J. and Hu, J. (2012) Synthesis and Ag-Content-Depended Electrochemical Properties of Ag/ZnO Heterostructured Nanomaterials. Materials Letters, 79, 205-208. https://doi.org/10.1016/j.matlet.2012.03.098

[11] Nam, H. J., Yi, G., Jeong, S., Boo, J. and Jung, D. (2009) Fabrication of NanoStructured Hemispheres and Nanopillars Using Laterally Migrating Polymer Templates. Journal of Nanoscience and Nanotechnology, 9, 6008-6012. https://doi.org/10.1166/jnn.2009.1233

[12] Gong, P., Li, H., He, X., Wang, K., Hu, J., Zhang, S. and Yang, X. (2007) Preparation and Antibacterial Activity of Fe3O4@Ag Nanoparticles. Nanotechnology, 18, 285604. https://doi.org/10.1088/0957-4484/18/28/285604

[13] Durán, N., Marcarto, P.D., De Souza, G.I.H., Alves, O.L. and Esposito, E. (2007) Antibacterial Effect of Silver Nanoparticles Produced by Fungal Process on Textile Fabrics and Their Effluent Treatment. Journal of Biomedical Nanotechnology, 3, 203-208. https://doi.org/10.1166/jbn.2007.022

[14] Rogozea, E.A., Olteanu, N.L., Crisciu, A.V., Petcu, A.R. and Mihaly, M. (2014) Highly Homogeneous Nanostructured Templates Based on Environmental Friendly Microemulsion for Nanomaterials Processing. Materials Letters, 132, 346-348. https://doi.org/10.1016/j.matlet.2014.06.072

[15] Yuan, P. and Bao, X. (2013) A Facile and Finely-Controlled Synthesis of Versatile Porous Silica Nanomaterials: Nanopods, Helical Nanorods and Nanovesicles. Materials Letters, 109, 199-202. https://doi.org/10.1016/j.matlet.2013.05.086

[16] Bae, C.H., Nam, S.H. and Park, S.M. (2002) Formation of Silver Nanoparticles by Laser Ablation of a Silver Target in $\mathrm{NaCl}$ Solution. Applied Surface Science, 197-198, 628-634. https://doi.org/10.1016/S0169-4332(02)00430-0

[17] Qazi, U.Y., Kajimoto, S. and Fukumura, H. (2014) Effect of Sodium Dodecyl Sulfate on the Formation of Silver Nanoparticles by Biphotonic Reduction of Silver Nitrate in Water. Chemistry Letters, 43, 1693-1695. https://doi.org/10.1246/cl.140617

[18] Nakamura, T., Magara, H., Herbani, H. and Sato, S. (2011) Fabrication of Silver Nanoparticles by Highly Intense Laser Irradiation of Aqueous Solution. Applied Physics A, 104, 1021-1024. http://dx.doi.org/10.1007/s00339-011-6499-5

[19] Abid, J.P., Wark, A.W., Brevet, P.F. and Girault, H.H. (2002) Preparation of Silver Nanoparticles in Solution from a Silver Salt by Laser Irradiation. Chemical Communications, 7, 792-793. https://doi.org/10.1039/b200272h

[20] Kamat, P.V., Flumiani, M. and Hartland, G.V. (1998) Picosecond Dynamics of Silver Nanoclusters. Photoejection of Electrons and Fragmentation. The Journal of Physical Chemistry B 102, 3123-3128. https://doi.org/10.1021/jp980009b

[21] Heilmann, A., Kiesow, A., Gruner, M. and Kreibig, U. (1999) Optical and Electrical Properties of Embedded Silver Nanoparticles at Low Temperatures. Thin Solid Films, 343-344, 175-178. https://doi.org/10.1016/S0040-6090(98)01599-5

[22] Ogawa, T., Yanai, N., Monguzzi, A. and Kimizuka, N. (2015) Highly Efficient Photon Upconversion in Self-Assembled Light-Harvesting Molecular Systems. Scientific Reports, 5, 10882. https://doi.org/10.1038/srep10882 